\title{
Enteroendocrine $K$ and $L$ cells in healthy and type 2 diabetic individuals
}

\author{
Tina Jorsal $^{1}$ • Nicolai A. Rhee ${ }^{1,2}$ - Jens Pedersen ${ }^{3,4}$ - Camilla D. Wahlgren ${ }^{1}$. \\ Brynjulf Mortensen ${ }^{1,5}$ • Sara L. Jepsen ${ }^{3,4}$ • Jacob Jelsing ${ }^{6}$ Louise S. Dalbøge ${ }^{6,7}$. \\ Peter Vilmann ${ }^{8,9}$ • Hazem Hassan ${ }^{8,9}$ • Jakob W. Hendel ${ }^{8,9}$ • Steen S. Poulsen ${ }^{3,4}$. \\ Jens J. Holst ${ }^{3,4}$ • Tina Vilsbøll ${ }^{1,10,11}$ - Filip K. Knop ${ }^{1,4,10}$
}

Received: 18 April 2017 / Accepted: 14 August 2017 /Published online: 28 September 2017

(C) Springer-Verlag GmbH Germany 2017

\begin{abstract}
Aims/hypothesis Enteroendocrine $\mathrm{K}$ and $\mathrm{L}$ cells are pivotal in regulating appetite and glucose homeostasis. Knowledge of their distribution in humans is sparse and it is unknown whether alterations occur in type 2 diabetes. We aimed to evaluate the distribution of enteroendocrine $\mathrm{K}$ and $\mathrm{L}$ cells and relevant prohormone-processing enzymes (using immunohistochemical staining), and to evaluate the mRNA expression of the corresponding genes along the entire intestinal tract in individuals with type 2 diabetes and healthy participants.

Methods In this cross-sectional study, 12 individuals with type 2 diabetes and 12 age- and BMI-matched healthy individuals underwent upper and lower double-balloon enteroscopy with mucosal biopsy retrieval from approximately every $30 \mathrm{~cm}$ of the small intestine and from seven specific anatomical locations in the large intestine.

Results Significantly different densities for cells positive for chromogranin A (CgA), glucagon-like peptide-1, glucose-
\end{abstract}

Electronic supplementary material The online version of this article (https://doi.org/10.1007/s00125-017-4450-9) contains peer-reviewed but unedited supplementary material, which is available to authorised users.

Filip K. Knop

filipknop@dadlnet.dk

1 Center for Diabetes Research, Gentofte Hospital, University of Copenhagen, Kildegårdsvej 28, DK-2900 Hellerup, Denmark

2 Present address: Novo Nordisk A/S, Bagsværd, Denmark

3 Department of Biomedical Sciences, Faculty of Health and Medical Sciences, University of Copenhagen, Copenhagen, Denmark

4 Novo Nordisk Foundation Center for Basic Metabolic Research, Faculty of Health and Medical Sciences, University of Copenhagen, Copenhagen, Denmark

5 Present address: Chr. Hansen A/S, Hørsholm, Denmark

dependent insulinotropic polypeptide, peptide YY, prohormone convertase (PC) $1 / 3$ and PC2 were observed along the intestinal tract. The expression of $C H G A$ did not vary along the intestinal tract, but the mRNA expression of GCG, GIP, PYY, $P C S K 1$ and $P C S K 2$ differed along the intestinal tract. Lower counts of CgA-positive and $\mathrm{PC} 1 / 3$-positive cells, respectively, were observed in the small intestine of individuals with type 2 diabetes compared with healthy participants. In individuals with type 2 diabetes compared with healthy participants, the expression of $G C G$ and $P Y Y$ was greater in the colon, while the expression of GIP and PCSK1 was greater in the small intestine and colon, and the expression of PCSK2 was greater in the small intestine.

Conclusions/interpretation Our findings provide a detailed description of the distribution of enteroendocrine $\mathrm{K}$ and $\mathrm{L}$ cells and the expression of their products in the human intestinal tract and demonstrate significant differences between individuals with type 2 diabetes and healthy participants.

Present address: Novo Nordisk Research Center, Seattle, WA, USA

8 Endoscopic Unit, Gentofte Hospital, University of Copenhagen, Hellerup, Denmark

9 Present address: Gastrounit, Herlev and Gentofte Hospital, University of Copenhagen, Herlev, Denmark

10 Department of Clinical Medicine, Faculty of Health and Medical Sciences, University of Copenhagen, Copenhagen, Denmark

11 Steno Diabetes Center Copenhagen, University of Copenhagen, Gentofte, Denmark 
Trial registration: NCT03044860.

Keywords Chromogranin A · Double-balloon enteroscopy · Enteroendocrine cells · Glucagon-like peptide-1 .

Glucose-dependent insulinotropic polypeptide .

Immunohistochemistry $\cdot$ mRNA expression · Peptide YY .

Prohormone convertase $\cdot$ Type 2 diabetes

$\begin{array}{ll}\text { Abbreviations } \\ \text { CgA } & \text { Chromogranin A } \\ \text { DBE } & \text { Double-balloon enteroscopy } \\ \text { GIP } & \text { Glucose-dependent insulinotropic polypeptide } \\ \text { GLP-1 } & \text { Glucagon-like peptide-1 } \\ \text { NAPS } & \text { Nurse-administered propofol sedation } \\ \text { PC1/3 } & \text { Prohormone convertase } 1 / 3 \\ \text { PC2 } & \text { Prohormone convertase } 2 \\ \text { PYY } & \text { Peptide YY } \\ \text { qPCR } & \text { Quantitative PCR }\end{array}$

\section{Introduction}

Enteroendocrine cells and their secretory products have turned out to constitute important players in the regulation of glucose homeostasis and appetite [1-4]. Some of the most exhaustively studied gut hormones are the so-called incretin hormones: glucose-dependent insulinotropic polypeptide (GIP) from enteroendocrine $\mathrm{K}$ cells, and glucagon-like peptide-1 (GLP-1) from enteroendocrine L cells [5, 6]. Owing to their insulinotropic effects, GIP and GLP-1 are responsible for up to $70 \%$ of insulin release after an oral glucose challenge, i.e. the incretin effect. Impairment or absence of the incretin effect is observed in type 2 diabetes [7], and several GLP-1-based treatments have been developed and are now available for the management of type 2 diabetes [8].

Despite the comprehensive expansion of knowledge within the incretin field that has happened over the last few decades, the precise distribution of $\mathrm{K}$ and $\mathrm{L}$ cells and the regional expression of their hormonal products in man are uncertain. Existing assumptions about the distribution of $\mathrm{K}$ and $\mathrm{L}$ cells in the human gut are based on findings from animal studies and a few human studies with notable limitations: investigations were performed in limited parts of the gut, and samples were typically obtained from individuals who underwent abdominal surgery or had biopsy retrieval due to gastrointestinal pathology [9-12]. Thus, the distribution of enteroendocrine $\mathrm{K}$ and $\mathrm{L}$ cells and expression of their hormonal products have never been investigated in the entire human gut of living healthy individuals or compared with individuals with type 2 diabetes in a systematically and standardised fashion. Furthermore, the expression pattern of the processing enzyme, prohormone convertase (PC) $1 / 3$, which is responsible for the formation of GIP and GLP-1 from their precursors (proGIP in $\mathrm{K}$ cells and proglucagon in $\mathrm{L}$ cells) [13], and the distribution of PC1/3-positive cells along the intestinal tract remain unknown. It is well known that PC2 cleavage sites are present in proGIP and proglucagon [13], but, as for PC1/3, the intestinal expression pattern of $\mathrm{PC} 2$ and distribution of $\mathrm{PC} 2$-positive cells in the intestine have not been determined.

The development of double-balloon enteroscopy (DBE) has made it possible to access the entire gastrointestinal tract in living individuals and retrieve biopsies of high quality with limited invasiveness. Using anterograde and retrograde DBE with frequent biopsy sampling along the entire intestinal tract (around 3000 biopsies in total), we evaluated the distribution of enteroendocrine $\mathrm{K}$ and $\mathrm{L}$ cells and the expression of their hormonal products in 12 healthy human participants. To investigate whether differences with respect to the distribution and hormone products of these cells could play a role in the pathophysiology of type 2 diabetes, we also studied 12 ageand BMI-matched participants who had been diagnosed with type 2 diabetes.

\section{Methods}

The study was conducted according to the Helsinki Declaration, Seventh Revision 2013 (all participants gave informed consent). It was registered at Clinicaltrials.gov (NCT03044860) and the Danish Data Protection Agency, and approved by the Scientific-Ethical Committee of the Capital Region of Denmark (reg. no. H-3-2010-115).

Participants Twelve individuals diagnosed with type 2 diabetes (recruited via the diabetes outpatient clinic at Gentofte Hospital) and 12 non-diabetic healthy individuals matched for age and BMI (recruited via the website forsoegsperson.dk or through contact established in previous study participation at the research department) were included in the study. Basic demographic details are shown in Table 1. For individuals with type 2 diabetes, the inclusion criteria were diagnosis of type 2 diabetes (at least 3 months prior to study inclusion), treatment with diet counselling alone or combined with metformin or sulfonylurea, white ethnicity, age $>25$ and $<70$ years, and negativity for autoantibodies to GAD and islet cell autoantibodies. The exclusion criteria included treatment with dipeptidyl peptidase 4 inhibitors or medicine that could not be withheld for $12 \mathrm{~h}, \mathrm{BMI}>35 \mathrm{~kg} / \mathrm{m}^{2}$ and any other condition that would contraindicate propofol sedation or enteroscopy. For healthy individuals, inclusion criteria included normal fasting plasma glucose and oral glucose tolerance, white ethnicity and age $>25$ and $<70$ years. The exclusion criteria were identical to those for participants with type 2 diabetes with the addition of first-degree relative(s) with type 1 or type 2 diabetes. 
Table 1 Demographics of participants with type 2 diabetes and healthy individuals

\begin{tabular}{llll}
\hline Variable & Type 2 diabetes & Healthy & $p$ value \\
\hline Sex $(\mathrm{M} / \mathrm{F})$ & $9 / 3$ & $8 / 4$ & - \\
Age $($ years $)$ & $51(34-63)$ & $50(41-67)$ & 0.66 \\
$\mathrm{BMI}\left(\mathrm{kg} / \mathrm{m}^{2}\right)$ & $26.8(23.7-31.5)$ & $27.1(20.3-30.8)$ & 0.92 \\
$\mathrm{HbA}_{1 \mathrm{c}}(\%)$ & $6.5(5.4-9.9)$ & $5.3(4.8-6.1)$ & - \\
$\mathrm{HbA}_{1 \mathrm{c}}(\mathrm{mmol} / \mathrm{mol})$ & $48(36-85)$ & $34(29-43)$ & - \\
Duration of type 2 diabetes (years) & $5.0(1.0-9.0)$ & - & - \\
\hline
\end{tabular}

Data are means (ranges)
Experimental procedures After a screening visit, the participants underwent two procedure days at Gentofte Hospital. Glucose-lowering drugs, if any, were withheld for 1 week before each of the two study days.

First, an anterograde DBE was performed under nurseadministered propofol sedation (NAPS). The DBE scope was an EN-450 T5 from Fujinon (Saitama, Japan). DBE enables deep intubation of the gastrointestinal tract by a push-and-pull approach. When progression was no longer possible due to pressure from accumulated intestine, an ink mark was placed submucosally to indicate the maximal depth of insertion. Using biopsy forceps, two mucosal biopsies were retrieved at approximately $30 \mathrm{~cm}$ intervals during scope retraction as judged visually by the endoscopist. Biopsies retrieved from the duodenum, the ligament of Treitz area and the ileocaecal transition were distributed to tubes marked corresponding to these anatomically specific regions. The variable number of biopsy 'stations' (7-22) in the jejunum and proximal ileum were divided equally into seven groups (nos 3-9, Fig. 1). When several biopsies were obtained within the same region, the mean of the biopsy data was calculated.

On a separate day, retrograde DBE during NAPS was performed. Intubation was conducted until the ink mark was visualised (as an indication of total enteroscopy) or when time issues occurred [14]. Next, biopsies were collected from six anatomically specific sites in the large intestine (caecum, ascending colon, transverse colon, descending colon, sigmoid colon and rectum; Fig. 1). If the submucosal ink mark was not identified during retrograde enteroscopy, an area between the anterograde and the retrograde enteroscopies was by definition termed 'not biopsied' (Fig. 2). The DBE procedures and biopsy sampling have previously been described in detail by Rhee et al [14].

Gene expression analysis The mRNA expression of the genes of interest CHGA, GIP, GCG, PYY, PCSK1 and $P C S K 2$ genes as well as the genes used for normalisation RPS18, ACTB, GADPH, HPRT1, RPL13, SDHA, TBP, $Y W H A Z$ and $H M B S$ were investigated. One biopsy sample from each biopsy site was immediately incubated in RNAlater solution (to preserve mRNA quality) (Sigma Aldrich R0901, MO, USA). Subsequently, standard RNA purification, cDNA synthesis and quantitative PCR (qPCR) analysis were performed. See ESM Methods for further details.

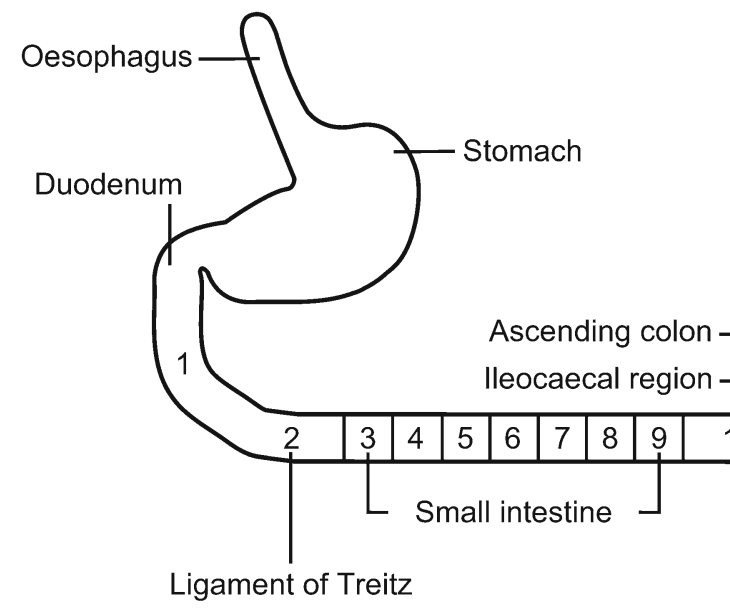

Fig. 1 The gastrointestinal tract and biopsy sampling sites. Biopsies were sampled from nine anatomically well-defined areas: the duodenum (1), the area around the ligament of Treitz (2), the ileocaecal region (10), caecum (11), ascending colon (12), transverse colon (13), descending

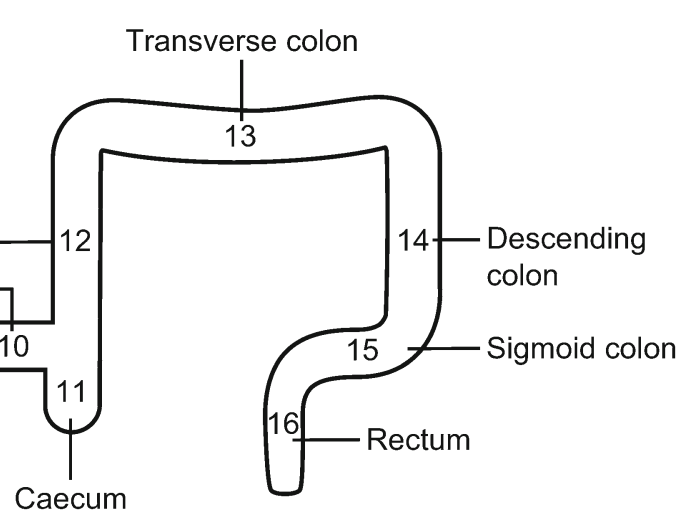

colon (14), sigmoid colon (15) and rectum (16). Furthermore, biopsies were taken every $30 \mathrm{~cm}$ in the small intestine and divided equally into seven groups (3-9) 


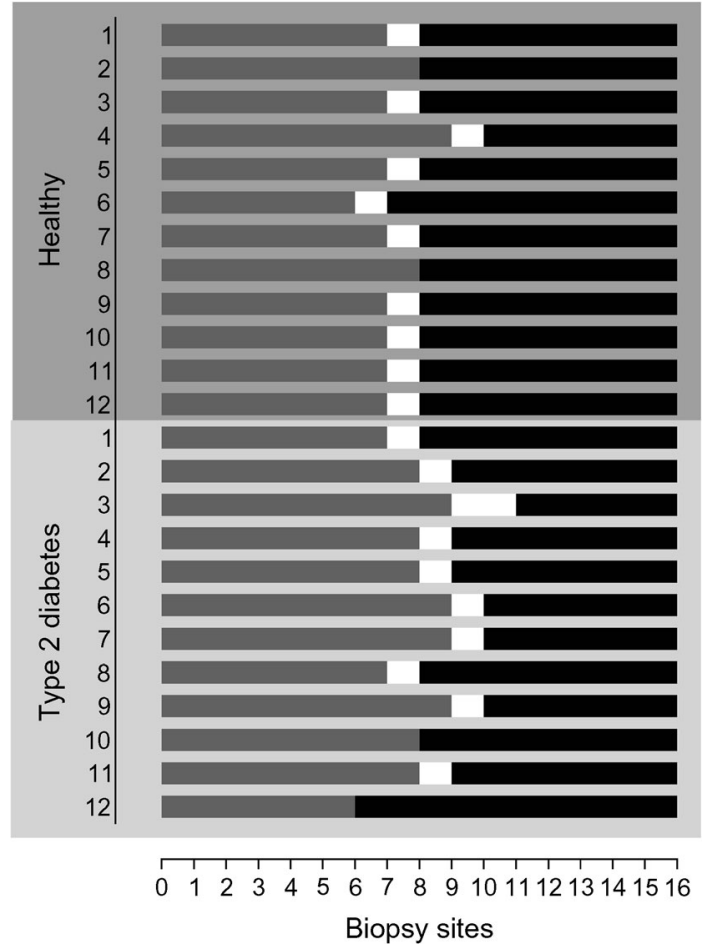

Fig. 2 Biopsy samples obtained along the gut. The horizontal axis represents biopsy sites along the gut, with numbers according to Fig. 1. The vertical axis represents the individual participants with type 2 diabetes as one group $(n=12)$ and the individual healthy participants as another group $(n=12)$. Grey and black bars represent the length of anterograde and retrograde enteroscopy, respectively. White bars represent an unknown length of unexamined intestine. Enteroscopy and biopsy sampling of the entire intestinal tract was possible in two healthy individuals (nos 2 and 8 ) and two participants with type 2 diabetes (nos 10 and 12)

Immunohistochemistry The other biopsy sampled from each site was immediately fixated and then embedded in paraffin, cut in thin slide sections and dewaxed. Subsequently, antigen retrieval was performed, with incubations with (1) specific primary antibodies, (2) a second layer of antibodies and (3) a third layer of an avidin-biotin complex. Finally, counterstaining was performed, generating biopsy slides with cells positive for chromogranin A (CgA), GIP, peptide YY (PYY), GLP-1, PC1/3 and PC2, respectively. See ESM Methods for further details.

Cell count The distribution of enteroendocrine cells was evaluated on representative biopsy slide sections based on immunohistochemical staining. Using the newCAST system (Visiopharm, Hørsholm, Denmark), the number of all immunopositive (stained) cells within the epithelial area was counted and divided by the size of the epithelial area. See ESM Methods for further details.

Statistics Student's $t$ test was used to evaluate age and BMI match between groups. Gene expression data were calculated according to the efficiency-corrected formula using an internal calibrator as reference [15] and are presented as $\log _{2}$ transformed data centred on the mean of duodenum in the healthy group. Expression data are presented as medians (lines) with 1st and 3rd quartiles (boxes), 5th and 95th percentiles (whiskers) and means (dots). Immunohistochemical cell quantification data are presented as means with SEM. Biopsy slides that presented with no positively stained cells were included in the statistical analysis as 0 (zero) cells. All data were statistically processed using SAS software version 9.1 (SAS Institute, Cary, NC, USA). Small intestine (Fig. 1, regions 1-10) and colon (Fig. 1, regions 11-16) were assessed separately using two-way mixed-model ANOVA to evaluate the main effects of intestinal localisation, group, and localisation $\times$ group interaction. Significant interactions were evaluated post hoc using Student's unpaired $t$ tests comparing the difference $(\Delta)$ in most proximal vs most distal biopsy (for small intestine: duodenum vs ileocaecal region) between the two groups. Statistical significance was accepted at $p<0.05$. GraphPad Prism Software version 7 (La Jolla, CA, USA) was used to create figures.

\section{Results}

Total enteroscopy with visualisation of the ink mark and biopsy sampling from the entire intestinal tract was possible in four of the 24 participants: two with type 2 diabetes and two healthy individuals (Fig. 2). The majority of the intestinal tract was visualised and biopsied in the remaining 20 participants, judging from the depth of endoscope insertion (the mean depth of bowel insertion for all participants was $471 \mathrm{~cm}$ anterograde and $218 \mathrm{~cm}$ retrograde [14]). No sign of pathology was observed during the gastrointestinal investigation of the 24 participants except that two participants (both healthy) were found to have Encheliophis vermicularis and a single polyp was found in another two participants (one in each group).

CgA The relative mRNA expression levels of $C H G A$ are illustrated in Fig. 3a. Expression levels were similar along the intestinal tract, and no differences were observed between the two groups. By immunohistochemistry, clear regional differences in the density (cells $/ \mathrm{mm}^{2}$ epithelium) of $\mathrm{CgA}$ positive cells were observed along the intestinal tract: a drop in density of $\mathrm{CgA}$-positive cells along the small intestine $(p<0.0001)$ and an increase $(p<0.0001)$ along the colon (Fig. 3b). A greater density of CgA-positive cells was found in the small intestine of healthy individuals than participants with type 2 diabetes $(p=0.006)$.

GIP A drop in the expression of GIP along the small intestine $(p<0.0001)$ was observed. Greater expression of GIP was 
a

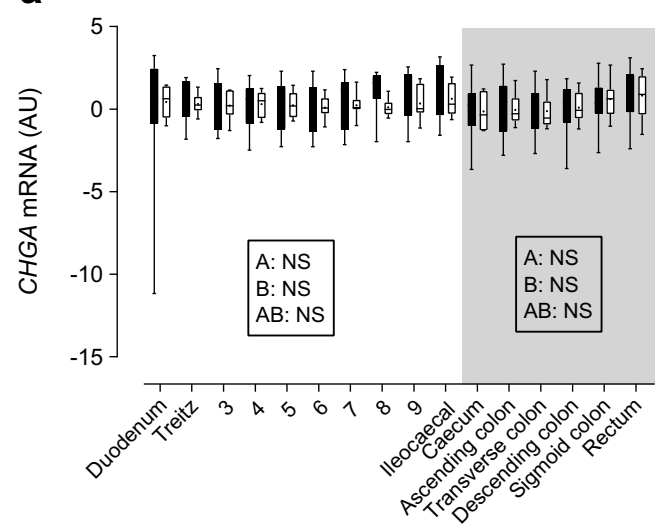

b

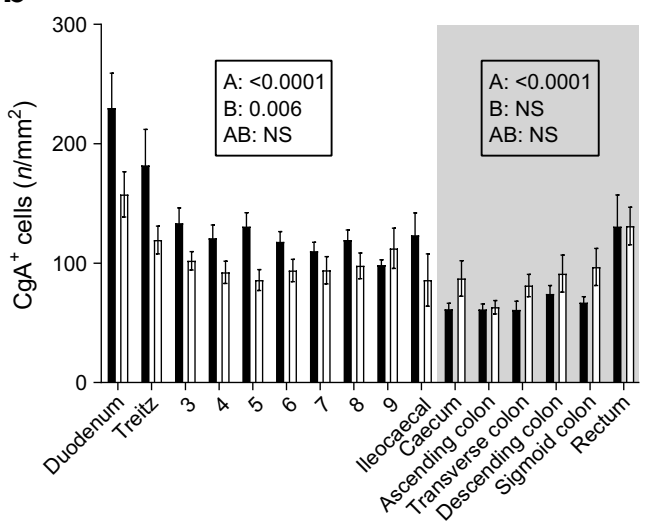

C

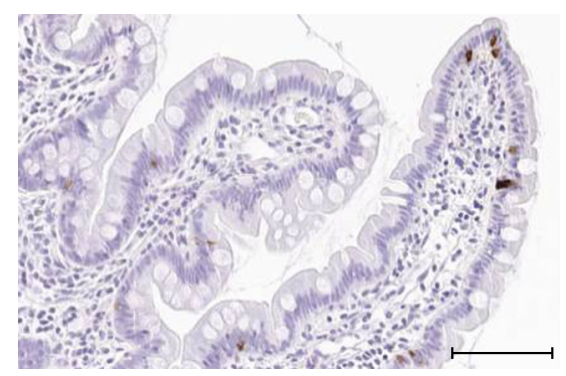

Fig. $3 \mathrm{CgA}$ in the gut. White bars, participants with type 2 diabetes $(n=12)$; black bars, healthy individuals $(n=12)$. Numbers $3-9$ represent small intestinal samples. Two-way mixed-model ANOVA was applied to the main effects of: $\mathrm{A}$, intestinal localisation; $\mathrm{B}$, group; $\mathrm{AB}$, localisation $\times$ group interaction. (a) mRNA expression levels of $C H G A$ in arbitrary units (AU) (values are $\log _{2}$-transformed and centred on the mean of the duodenum in the healthy group). Data are medians (lines) with 1 st and 3 rd quartiles (boxes), 5th and 95th percentiles (whiskers) and means (dots). (b) Density (cells $/ \mathrm{mm}^{2}$ epithelium) of CgA-positive cells (data presented as mean \pm SEM). (c) Histology shows immunopositive cells stained for $\mathrm{CgA}$ in a small intestinal sample. Scale bar, $50 \mu \mathrm{m}$

seen in both the small intestine $(p=0.002)$ and colon $(p=0.023)$ of participants with type 2 diabetes compared with healthy individuals (Fig. 4a). Similar to the expression pattern of GIP, we observed a decline in the density of GIP-positive cells (as assessed by immunohistochemistry) along the small intestine $(p<0.0001)$ (Fig. 4b). a

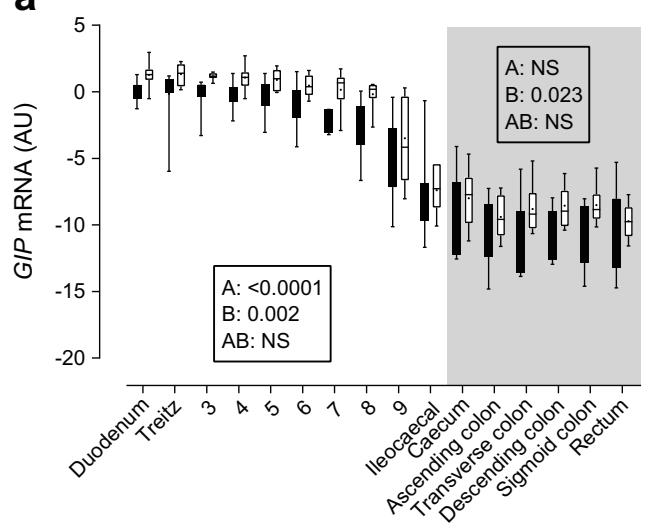

b

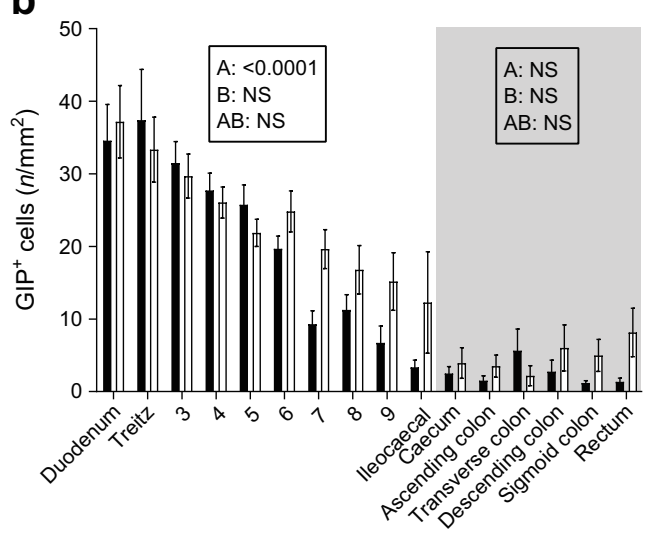

C

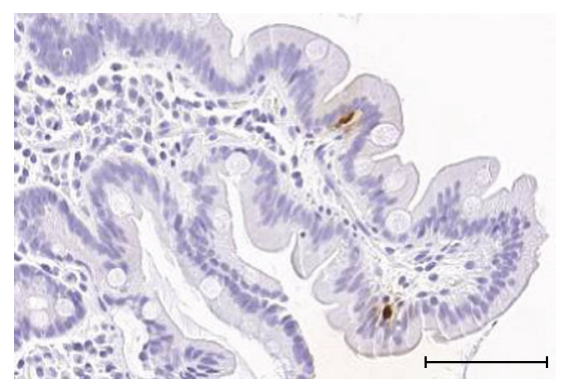

Fig. 4 GIP in the gut. White bars, participants with type 2 diabetes $(n=12)$; black bars, healthy individuals $(n=12)$. Numbers $3-9$ represent small intestinal samples. Two-way mixed-model ANOVA was applied to the main effects of: A, intestinal localisation; B, group; AB, localisation $\times$ group interaction. (a) mRNA expression levels of GIP in arbitrary units (AU) (values are $\log _{2}$-transformed and centred on the mean of the duodenum in the healthy group. Data are medians (lines) with 1st and 3rd quartiles (boxes), 5th and 95th percentiles (whiskers) and means (dots). (b) Density (cells $/ \mathrm{mm}^{2}$ epithelium) of GIP-positive cells (data presented as mean $\pm \mathrm{SEM}$ ). (c) Histology shows immunopositive cells stained for GIP in a duodenal sample. Scale bar, $50 \mu \mathrm{m}$

GLP-1 As illustrated in Fig. 5a, there was an increasing expression of $G C G$ along the small intestine $(p<0.0001)$ and colon $(p<0.0001)$. A greater expression of $G C G$ was observed in the colon of participants with type 2 diabetes compared with healthy individuals $(p=0.0009)$. Interaction 
Fig. 5 GLP-1 and PYY in the gut. White bars, participants with type 2 diabetes $(n=12)$; black bars, healthy individuals $(n=12)$. Numbers 3-9 represent small intestinal samples. Two-way mixed-model ANOVA was applied to the main effects of: A, intestinal localisation; B, group; $\mathrm{AB}$ : localisation $\times$ group interaction. (a) mRNA expression levels of $G C G$ in arbitrary units (AU) (values are $\log _{2^{-}}$ transformed and centred on the mean of the duodenum in the healthy group. Data are medians (lines) with 1 st and 3 rd quartiles (boxes), 5th and 95th percentiles (whiskers) and means (dots). (b) Density (cells $/ \mathrm{mm}^{2}$ epithelium) of GLP-1-positive cells (data presented as mean $\pm \mathrm{SEM}$ ). (c) Histology shows immunopositive cells stained for GLP-1 in a colon sample. Scale bar, $25 \mu \mathrm{m}$. (d) mRNA expression level of $P Y Y$. (e) Density of PYY-positive cells. (f) Histology shows

immunopositive cells stained for PYY in a rectal sample. Scale bar, $50 \mu \mathrm{m}$
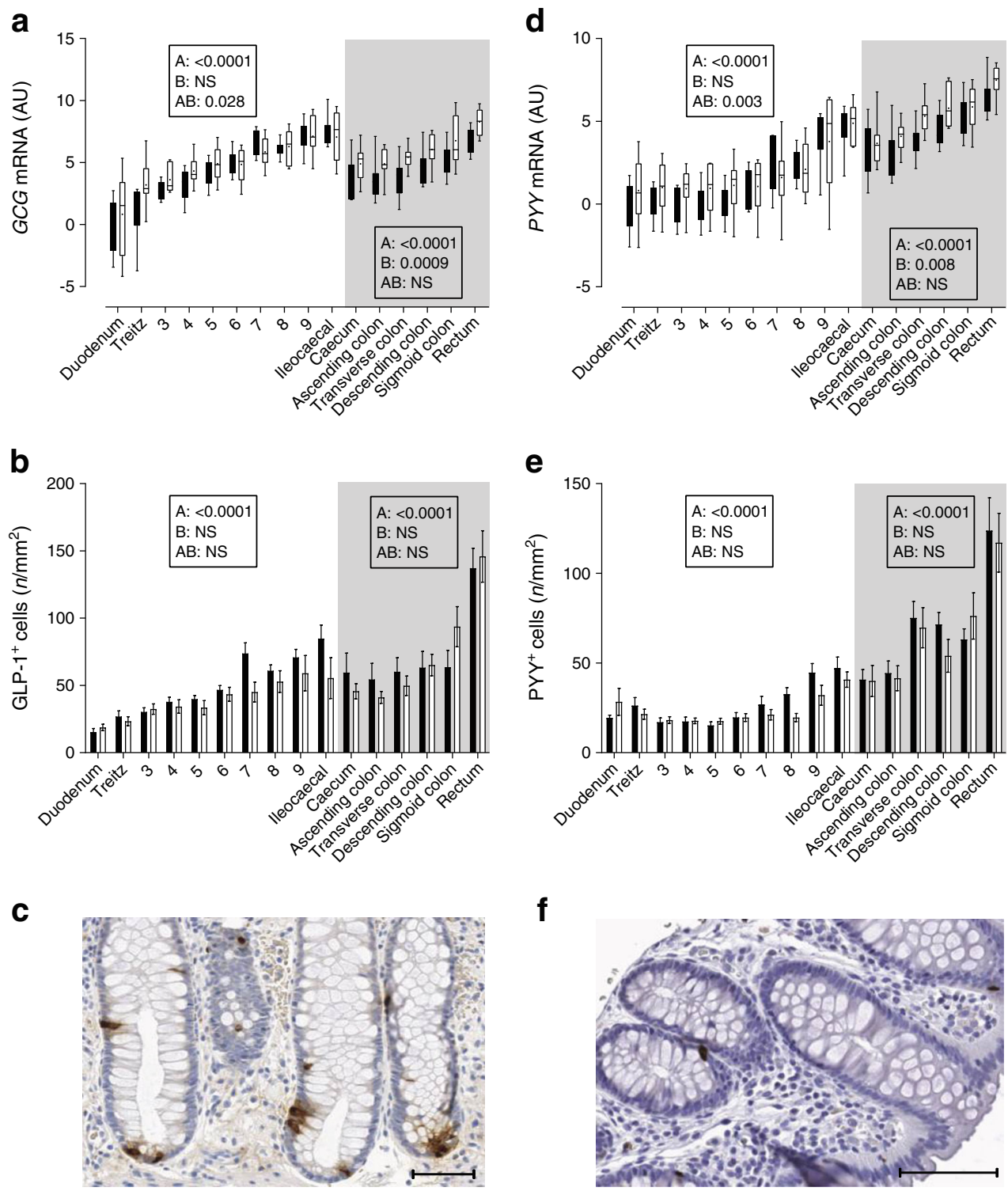

f



was observed for small intestinal $G C G$ expression $(p<0.028)$ (Fig. 5a). Post hoc $t$ tests showed that $\Delta$ (proximal duodenum vs distal ileocaecal biopsy) $G C G$ expression tended to be greater in healthy individuals compared with those who had type 2 diabetes $(p=0.050)$. Similar to the expression pattern of $G C G$, an increasing density of GLP-1-positive cells (as assessed by immunohistochemistry) along the small intestine $(p<0.0001)$ and along the colon $(p<0.0001)$ was observed (Fig. 5b).

PYY As depicted in Fig. 5d, an increasing expression of $P Y Y$ was observed along the small intestine $(p<0.0001)$ and along the colon $(p<0.0001)$. Greater PYY expression was observed in the colon in type 2 diabetes compared with healthy individuals $(p=0.008)$. Interaction was observed for small intestinal PYY expression $(p<0.003)$ (Fig. 5d). Post hoc $t$ tests showed that $\Delta$ (proximal duodenum vs distal ileocaecal biopsy) $P Y Y$ expression was non-significantly greater in healthy individuals than participants with type 2 diabetes $(p<0.07)$. By immunohistochemistry, we observed increasing density of PYY-positive cells along the small intestine $(p<0.0001)$ and along the colon $(p<0.0001)$ (Fig. 5e).

PC1/3 Changes in the expression of PCSK1 along the small intestine $(p=0.008)$ and colon $(p<0.0001)$ were observed. Expression of PCSK1 was greater in the small intestine $(p=0.0001)$ and the colon $(p=0.0004)$ of type 2 diabetes patients compared with healthy individuals (Fig. 6a). By immunohistochemistry, we observed decreasing densities of PC1/3-positive cells along the small intestine $(p<0.0001)$ and increasing densities along the colon $(p<0.0001)$ in both groups. Lower densities of PC1/3-positive cells were observed in the small intestine of participants with type 2 diabetes compared with healthy individuals $(p=0.036)$ (Fig. 6b). 
Fig. $6 \mathrm{PC} 1 / 3$ and $\mathrm{PC} 2$ in the gut. White bars, participants with type 2 diabetes $(n=12)$; black bars, healthy individuals $(n=12)$. Numbers 3-9 represent small intestinal samples. Two-way ANOVA was applied to the main effects of: A, intestinal localisation; $\mathrm{B}$, group; $\mathrm{AB}$, localisation $\times$ group interaction. (a) mRNA expression levels of PCSK1 in arbitrary units (AU) (values are $\log _{2}$-transformed and centred on the mean of the duodenum in the healthy group. Data are medians (lines) with 1st and 3 rd quartiles (boxes), 5 th and 95th percentiles (whiskers) and means (dots). (b) Density (cells $/ \mathrm{mm}^{2}$ epithelium) of $\mathrm{PC} 1 / 3$ positive cells (data presented as mean \pm SEM). (c) Histology shows immunopositive cells stained for $\mathrm{PC} 1 / 3$ in a small intestinal sample. Scale bar, $50 \mu \mathrm{m}$. (d) mRNA expression level of PCSK2. (e) Density of PC2-positive cells. (f) Histology shows immunopositive cells stained for PC2 in a small intestinal sample. Scale bar, $50 \mu \mathrm{m}$ a

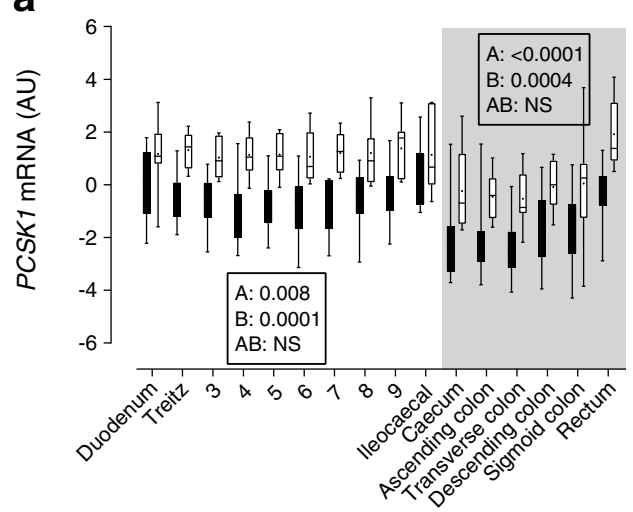

b

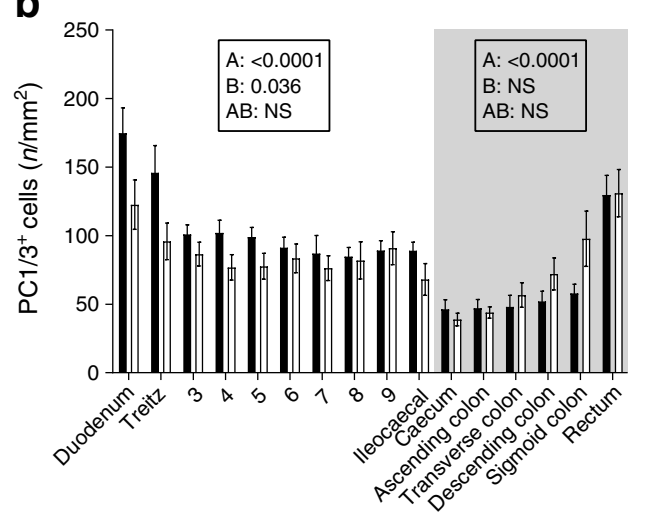

C



d

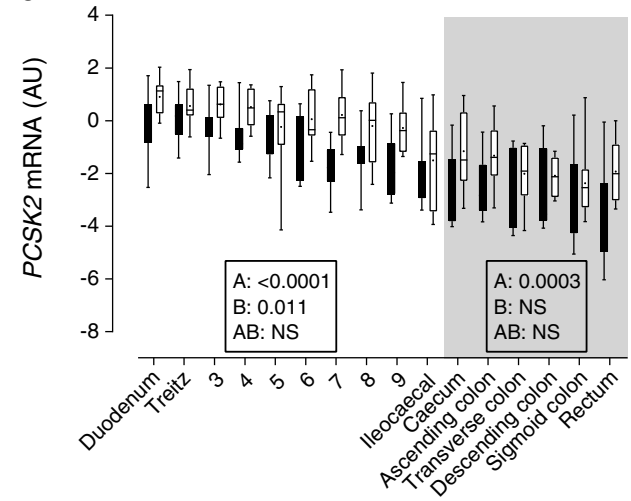

e

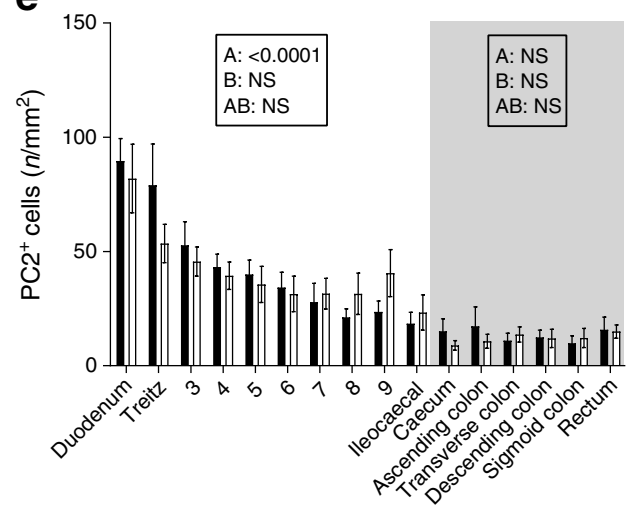

f

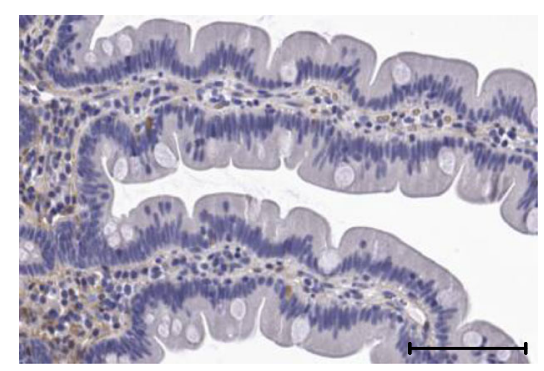

PC2 As illustrated in Fig. 6d, expression of PCSK2 was found throughout the intestine, decreasing along the small intestine $(p<0.0001)$ and colon $(p=0.0003)$. Greater expression of PCSK2 was observed in the small intestine of individuals with type 2 diabetes compared with healthy individuals $(p=0.011)$ (Fig. 6d). Decreasing densities of PC2-positive cells (as assessed by immunohistochemistry) were observed along the small intestine $(p<0.0001)$ (Fig. 6e).

\section{Discussion}

Using the DBE technique, we systematically collected biopsies from the entire human intestinal tract and characterised the distribution of enteroendocrine $\mathrm{K}$ and $\mathrm{L}$ cells and the expression of their hormonal products in healthy individuals and individuals with type 2 diabetes.

As type 2 diabetes implies overweight/obesity and dysfunctional glucose homeostasis that could correlate to changes in the enteroendocrine $\mathrm{K}$ and $\mathrm{L}$ cells, the study was designed to describe the distribution pattern of these cells and the expression of some of their products in the two prospectively recruited, similarly sized, well-defined and matched groups of volunteers without any known gastrointestinal disorders. Using DBE, it was possible to obtain a high number of samples throughout the intestinal tract: from nine anatomically specific regions (Fig. 1) and 7-22 'stations' along the jejunum and ileum. The biopsies from the anatomically welldefined areas were highly comparable. Greater uncertainty exists regarding biopsy locations in the jejunum and proximal ileum (biopsy locations 3-9, Fig. 1) owing to the variable 
length of intubation and hence the number of samples obtained between individuals. To deal with this issue, we systematically divided the jejunum and ileum into seven regions (see Methods). The submucosal ink mark placed at maximum depth of insertion during anterograde enteroscopy was seen during retrograde enteroscopy in four out of 24 individuals, indicating total enteroscopy (Fig. 2). It is assumed that the majority of the intestinal tract was investigated in the rest of the participants as judged by the length of the intubation achieved (for details, see our methodology paper [14]).

We used immunohistochemistry and cell count to quantify the enteroendocrine cells and prohormone-processing enzymes, and qPCR mRNA expression analysis to evaluate the hormonal/enzymatic products. Both methods are well established yet also pose limitations. A high specificity of antibodies used in immunohistochemistry is of great importance to ensure a minimum of unspecific staining (false positives). Accordingly, we chose antibodies that are well established and well-characterised [16]. Using immunohistochemistry and cell count from microphotographs of sliced biopsies, three-dimensional objects (i.e. enteroendocrine cells) are evaluated with a two-dimensional imaging technique. A more optimal technique for evaluation of cell distribution would be stereology, in which the true density (i.e. cells/ $\mathrm{mm}^{3}$ tissue) is estimated [17]. However, this technique would require complete transverse 'blocks' of tissue, which is incompatible with the high number of biopsy sites, and thus detailed mapping of the intestinal tract in living humans that was aimed for in the present study. Furthermore, the following should be taken into account. First, evaluation of mRNA expression indicates the activity of endocrine cells but does not provide a measure of the total production of a certain cell product as not all mRNA is translated into a final active product. Second, expression is relative since the specific mRNA transcripts are being related to the expression of a stably expressed gene (see ESM Methods for further details). Considering the biological heterogeneity of type 2 diabetes, more pronounced differences could potentially have been detected with a greater sample size and inclusion of patients with more extensive glucose dysregulation.

The work by Sjölund et al from 1983 on the distribution of enteroendocrine cells is the most detailed performed in human intestine so far using IHS with a wide array of antisera (25 types) against known or proposed gut neurohormonal peptides [9]. Minimally invasive techniques were not available at that time. Accordingly, samples were retrieved from only seven regions: proximal and distal duodenum, mid-jejunum, distal ileum, ascending colon, distal part of transverse colon or sigmoid colon and rectum. For each region, tissue material was obtained from 9-17 individuals. The samples were retrieved either during abdominal surgery (primarily performed due to malignancy) or during enteroscopic examinations involving biopsies in individuals with 'other gastrointestinal disorders', including a variety of unspecified symptoms and diseases (e.g. 'occult bleeding' and 'liver disease'). In 1985, Adrian et al used a radioimmunoassay technique to determine the amount of PYY in the gastric fundus and antrum, duodenum, jejunum, ileum, ascending colon, sigmoid colon and rectum [11]. Samples from each location were obtained from 5-8 individuals undergoing surgery due to carcinoma or gastric ulcers. In 1992, a study investigating the distribution of enteroendocrine $L$ cells in humans was reported by Eissele et al [10]. Samples were obtained from seven regions: duodenum, proximal and distal jejunum, ileum, ascending colon, transverse colon and rectum. The samples were obtained from only five participants who underwent surgery for carcinoma or Crohn's disease. In 2005, Guedes et al investigated the distribution of GIP-, GLP-1- and CgA-positive cells, respectively, using IHS on samples from every $20 \mathrm{~cm}$ of the small intestine in 30 human cadavers [18].

It should be emphasised that the four studies mentioned above investigated normal-appearing tissue samples with no sign of pathological changes. However, the presence of malignant or inflammatory changes in the intestinal area or the fast commencement of cell degradation post mortem [18, 19] could have influenced the general enteroendocrine cell distribution and function. Furthermore, the heterogeneity of the participants may have influenced the results.

In agreement with our results, Sjölund et al, Eissele et al, Adrian et al and Guedes et al, respectively, described variation in L cell products (GLP-1 and/or PYY) depending on gut localisation, with a higher density/amount in the distal jejunum and ileum compared with the duodenum and proximal jejunum [9-11, 18], and an increasing density/ amount from proximal to distal colon, with the highest levels in the rectum [9-11]. Our results support this L cell distribution pattern in healthy individuals and in type 2 diabetes, with increasing GCG and $P Y Y$ gene expression, as well as increasing density of GLP-1 and PYY-positive cells, along the small intestine and along the colon. We also observed the greatest signal of L cell markers (PYY- and GLP-1-positive cells and PYY mRNA expression) in the rectum, except for the expression of $G C G$. The implications - if any-of the observed differences between groups (significantly greater GCG and PYY expression in the colon of participants with type 2 diabetes compared with healthy individuals) are currently unknown. It is well established that the incretin effect is reduced in type 2 diabetes, and it has been proposed that a defect in nutrient-induced GLP-1 secretion may contribute to explain this phenomenon. However, studies investigating GLP-1 responses to nutrient stimuli in those with type 2 diabetes and non-diabetic individuals have shown that, in general, individuals with type 2 diabetes do not exhibit reduced plasma total GLP-1 responses [20, 21].

We observed a discrepancy between $G C G$ expression levels and density of GLP-1-positive cells along the gut. 
This emphasises that $\mathrm{L}$ cells in one part of the small intestine can behave differently from enteroendocrine L cells in another part of the small intestine, as suggested by Svendsen et al, who observed that the secretion pattern of $\mathrm{L}$ cells changes along the gastrointestinal tract in rats, i.e. that $\mathrm{L}$ cells secrete different ratios of PYY and GLP-1, with some secreting only GLP-1 [22]. In line with these findings, it is likely that more distally located $\mathrm{L}$ cells express proglucagon to a higher extent than more proximally located L cells.

Our findings of greater GIP expression and density of GIPpositive cells in the proximal part of the small intestine, both decreasing distally to the ileocaecal region in healthy individuals and those with type 2 diabetes, are in line with previous findings [9, 18]. Unlike Sjölund et al [9], who found GIP-positive cells to be absent from the large intestine, we were able to detect low levels of GIP-positive cells in the distal part of the intestinal tract. However, we cannot rule out that this is a result of unspecific antibody binding. However, we did observe mRNA expression of GIP in the colon of both groups, albeit at very low levels. The expression of GIP was significantly greater in individuals with type 2 diabetes along the entire intestinal tract. It could be speculated that the transcription of GIP is increased as a compensatory result of GIP resistance, i.e. the reduced insulinotropic effect of GIP observed in individuals with type 2 diabetes [23, 24]. In line with this, fasting GIP levels have been demonstrated to be higher in participants with type 2 diabetes compared with non-diabetic control participants $[25,26]$, and, furthermore, it has been proposed that GIP contributes to the hyperglycaemia seen in type 2 diabetes (primarily due to the glucagonotropic effect of GIP) [27]. However, data regarding GIP responses following oral glucose or mixed meals in individuals with type 2 diabetes have been inconsistent, and a systematic review with meta-analysis has suggested that postprandial GIP responses are similar in those with type 2 diabetes and healthy individuals [26].

Given that the acidic glycoprotein $\mathrm{CgA}$ is a component of cell vesicles and considered to play multiple roles in the secretory process of endocrine products, it is used as a general marker of enteroendocrine cells [28-30]. Unlike Guedes et al [18] who observed a constant density of CgA-positive cells along the small intestine, our study showed a significant decline. Furthermore, we observed a greater density of CgA-positive cells in the small intestine of healthy individuals than participants with type 2 diabetes. It could be speculated that the total number of $\mathrm{CgA}$ positive cells (enteroendocrine cells) is altered in type 2 diabetes - either as a consequence of the type 2 diabetic state or, perhaps, contributing to the pathogenesis of type 2 diabetes. We observed rather high numbers of $\mathrm{CgA}$ positive cells in the rectum of both groups. Recently, Engelstoft et al showed in mice that $\mathrm{CgA}$ was mainly localised to monoamine-secreting enteroendocrine cells and more sparsely to peptide-secreting enteroendocrine cells [31], perhaps supporting the proposal by Sjölund et al that the rectal enteroendocrine cells could have a primarily local (paracrine) function rather than systemic function [9].

Since $\mathrm{PC} 1 / 3$ is known to process prohormones leading to formation of GIP and GLP-1, respectively, we expected to find the presence of $\mathrm{PC} 1 / 3$ along the entire intestinal tract. We respectively observed a discrepancy involving greater expression of $P C S K 1 / 3$ and lower densities of $\mathrm{PC} 1 / 3$-positive cells in the small intestine of participants with type 2 diabetes compared with healthy participants. As outlined above, this finding should be interpreted with the view that some enteroendocrine cells can be very active in some regions and have low activity in other regions.

Since PC2 is primarily known for processing of proglucagon to glucagon in pancreatic alpha cells, it was interesting to find mRNA expression of PCSK2 and PC2positive cells along the entire intestinal tract. This could indicate that glucagon is produced in the gut, as recently suggested by Lund et al [32]. In line with this, it could be speculated that the greater PCSK2 expression in the small intestine of individuals with type 2 diabetes leads to formation of excess glucagon, contributing to type 2 diabetic hyperglucagonaemia. To further clarify this aspect, studies including immunohistochemical double-staining are warranted.

In conclusion, the present mapping of the distribution of the enteroendocrine $\mathrm{K}$ and $\mathrm{L}$ cells and the observed variations in expression levels of their related products along the human intestinal tract, combined with the demonstrated differences between participants with type 2 diabetes and healthy individuals, provide a reference work for scientists and clinicians. Combined with knowledge from physiological studies on circulating gut hormones, our data could be of value in understanding how the gut contributes to regulate glucose metabolism and appetite. Identification of PC2 in enteroendocrine cells is interesting because this might be consistent with formation of glucagon in the human gut. However, further studies are needed to prove this possibility and link our findings to the pathophysiology of type 2 diabetes.

Acknowledgements Special thanks to the study participants who volunteered for this study. The authors also thank A. Hammering, nurse-anaesthetist at Gentofte Hospital, University of Copenhagen, for performing NAPS during DBE, and H. M. Paulsen and L. Schorling Strange, biomedical laboratory technicians, NNF Center for Basic Metabolic Research, Faculty of Health and Medical Sciences, University of Copenhagen, for making biopsy slide sections and immunohistochemistry staining.

Data availability The raw data are available on request from the corresponding author. 
Funding This study was supported by Gentofte Hospital, University of Copenhagen and was partly supported by an unrestricted grant from the Novo Nordisk Foundation.

Duality of interest TJ, NAR, JP, CDW, BM, SLJ, JJ, LSD, PV, HH, JWH and SSP have no competing interests. Within the last 36 months, $\mathrm{JJH}$ has received lecture fees and/or unrestricted research grants from, participated on advisory boards of and/or consulted for AstraZeneca, GlaxoSmithKline, Intarcia, Hamni, MSD/Merck, Novartis, Novo Nordisk, Sanofi and Zealand Pharma. Within the last 36 months, TV has received lecture fees and/or unrestricted research grants from, participated on advisory boards of and/or consulted for Amgen, AstraZeneca, Boehringer Ingelheim, Eli Lilly, MSD/Merck, Novo Nordisk and Sanofi. Within the last 36 months, FKK has received lecture fees and/or unrestricted research grants from, participated on advisory boards of and/or consulted for Amgen, AstraZeneca, Boehringer Ingelheim, Eli Lilly, MedImmune, MSD/Merck, Novo Nordisk, Sanofi and Zealand Pharma.

Contribution statement TJ analysed and interpreted the data, performed statistical analysis and drafted the manuscript. NAR contributed to the study design, obtained funding, conducted experiments and critically revised the manuscript. JP acquired and analysed data and critically revised the manuscript. BM analysed data, performed statistical analysis and critically revised the manuscript. CDW, SLJ and LSD acquired data and critically revised the manuscript. SSP and JJ acquired data and performed study supervision and critical revision of the manuscript for important intellectual content. PV, HH and JWH conducted experiments and critically revised the manuscript. JJH acquired data and performed critical revision of the manuscript for important intellectual content. TV analysed and interpreted data and critically revised the manuscript. FKK designed and funded the study, analysed and interpreted data and drafted the manuscript together with TJ. All authors approved the final version for publication. FKK and TJ are the guarantors of this work and, as such, had full access to all the data in the study and take responsibility for the integrity of the data and the accuracy of the data analysis.

\section{References}

1. Rehfeld JF (1998) The new biology of gastrointestinal hormones. Physiol Rev 78:1087-1108

2. Patterson JT, Ottaway N, Gelfanov VM et al (2011) A novel human-based receptor antagonist of sustained action reveals body weight control by endogenous GLP-1. ACS Chem Biol 6:135-145

3. Svane MS, Jørgensen NB, Bojsen-Møller KN et al (2016) Peptide YY and glucagon-like peptide-1 contribute to decreased food intake after roux-en-Y gastric bypass surgery. Int J Obes 40:1699-1706

4. Gutzwiller J, Göke B, Drewe J et al (1999) Glucagonlike peptide-1: a potent regulator of food intake in humans. Gut BMJ 44:81-86

5. Meier JJ, Nauck MA, Schmidt WE, Gallwitz B (2002) Gastric inhibitory polypeptide: the neglected incretin revisited. Regul Pept 107:1-13

6. Holst JJ (2007) The physiology of glucagon-like peptide 1. Physiol Rev 87:1409-1439

7. Vilsbøll T, Holst JJ (2004) Incretins, insulin secretion and type 2 diabetes mellitus. Diabetologia 47:357-366

8. Lund A, Knop FK, Vilsboll T (2014) Glucagon-like peptide-1 receptor agonists for the treatment of type 2 diabetes: differences and similarities. Eur J Intern Med 25:407-414
9. Sjölund K, Sandén G, Håkanson R, Sundler F (1983) Endocrine cells in human intestine: an immunocytochemical study. Gastroenterology 85:1120-1130

10. Eissele R, Göke R, Willemer S et al (1992) Glucagon-like peptide-1 cells in the gastrointestinal tract and pancreas of rat, pig and man. Eur J Clin Investig 22:283-291

11. Adrian TE, Ferri G-L, Bacarese-Hamilton AJ, Fuessl HS, Polak JMBS (1985) Human distribution and release of a putative new gut hormone, peptide YY. Gastroenterology 89:1070-1077

12. Polak JM, Bloom SR, Kuzio M et al (1973) Cellular localization of gastric inhibitory polypeptide in the duodenum and jejunum. Gut 14:284-288

13. Ugleholdt R, Poulsen MLH, Holst PJ et al (2006) Prohormone convertase $1 / 3$ is essential for processing of the glucosedependent insulinotropic polypeptide precursor. J Biol Chem 281: 11050-11057

14. Rhee NA, Vilmann P, Hassan H et al (2014) The use of doubleballoon enteroscopy in retrieving mucosal biopsies from the entire human gastrointestinal tract. Scand J Gastroenterol 49:1143-1149

15. Pfaffl MW (2001) A new mathematical model for relative quantification in real-time RT-PCR. Nucleic Acids Res 29: 2003-2007

16. Rhee NA, Wahlgren CD, Pedersen J et al (2015) Effect of roux-en$\mathrm{Y}$ gastric bypass on the distribution and hormone expression of small-intestinal enteroendocrine cells in obese patients with type 2 diabetes. Diabetologia 58:2254-2258

17. Hansen CF, Vrang N, Torp Sangild P, Jelsing J (2013) Novel insight into the distribution of L-cells in the rat intestinal tract. Am J Trans Res 5:347-358

18. Guedes TP, Martins S, Costa M et al (2015) Detailed characterization of incretin cell distribution along the human small intestine. Surg Obes Relat Dis 11:1323-1331

19. Stan AD, Ghose S, Gao XM et al (2006) Human postmortem tissue: what quality markers matter? Brain Res 1123:1-11

20. Nauck MA, Vardarli I, Deacon CF et al (2011) Secretion of glucagon-like peptide-1 (GLP-1) in type 2 diabetes: what is up, what is down? Diabetologia 54:10-18

21. Calanna S, Christensen M, Holst JJ et al (2013) Secretion of glucagon-like peptide- 1 in patients with type 2 diabetes mellitus: systematic review and meta-analyses of clinical studies. Diabetologia 56:965-972

22. Svendsen B, Pedersen J, Albrechtsen NJW et al (2015) An analysis of cosecretion and coexpression of gut hormones from male rat proximal and distal small intestine. Endocrinology 156:847-857

23. Xu G, Kaneto H, Laybutt DR et al (2007) Downregulation of GLP-1 and GIP receptor expression by hyperglycemia. Diabetes 56:1551-1558

24. Vilsbøll T, Knop FK, Krarup T et al (2003) The pathophysiology of diabetes involves a defective amplification of the late-phase insulin response to glucose by glucose-dependent insulinotropic polypeptide - regardless of etiology and phenotype. J Clin Endocrinol Metab 88:4897-4903

25. Suh S, Kim MY, Kim SK et al (2016) Glucose-dependent insulinotropic peptide level is associated with the development of type 2 diabetes mellitus. Endocrinol Metab (Seoul) 31:134-141

26. Calanna S, Christensen M, Holst JJ et al (2013) Secretion of glucose-dependent insulinotropic polypeptide in patients with type 2 diabetes: systematic review and meta-analysis of clinical studies. Diabetes Care 36:3346-3352

27. Chia CW, Carlson OD, Kim W et al (2009) Exogenous glucosedependent insulinotropic polypeptide worsens postprandial hyperglycemia in type 2 diabetes. Diabetes 58:1342-1349

28. Facer P, Bishop AE, Lloyd RV, Wilson BS, Hennessy RJ, Polak JM (1985) Chromogranin: a newly recognized marker for endocrine cells of the human gastrointestinal tract. Gastroenterology 89: 1366-1373 
29. Rindi G, Leiter AB, Kopin AS, Bordi C, Solcia E (2004) The "normal" endocrine cell of the gut: changing concepts and new evidences. Ann N Y Acad Sci 1014:1-12

30. Mouland AJ, Bevan S, White JH, Hendy GN (1994) Human chromogranin a gene. J Biol Chem 269:6918-6926
31. Engelstoft MS, Lund ML, Grunddal KV et al (2015) A chromogranin A reporter for serotonin and histamine secreting enteroendocrine cells. Mol Endocrinol 29:1658-1671

32. Lund A, Bagger JI, Wewer Albrechtsen NJ et al (2016) Evidence of extrapancreatic Glucagon secretion in man. Diabetes 65:585-597 